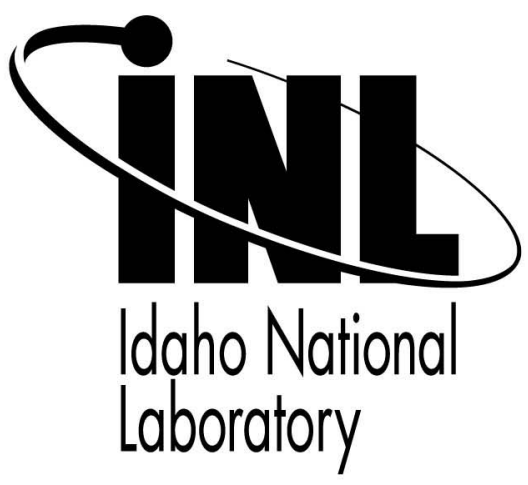

\title{
Issues of Mitigation Strategies in Augmented System for Next Generation Control Room
}

\author{
Joint $8^{\text {th }}$ IEEE HFPP $/ 13^{\text {th }}$ HPRCT \\ Tuan Q. Tran
}

August 2007

This is a preprint of a paper intended for publication in a journal or proceedings. Since changes may be made before publication, this preprint should not be cited or reproduced without permission of the author. This document was prepared as an account of work sponsored by an agency of the United States Government. Neither the United States Government nor any agency thereof, or any of their employees, makes any warranty, expressed or implied, or assumes any legal liability or responsibility for any third party's use, or the results of such use, of any information, apparatus, product or process disclosed in this report, or represents that its use by such third party would not infringe privately owned rights. The views expressed in this paper are not necessarily those of the United States Government or the sponsoring agency. 


\title{
Issues of Mitigation Strategies in Augmented System for Next Generation Control Room
}

\author{
Tuan Q. Tran \\ Idaho National Laboratory, PO Box 1625, Mail Stop 3605, Idaho Falls, ID 83415-3605, USA, \\ Email: \{Tuan.Tran\}@inl.gov
}

\begin{abstract}
Past research on augmented systems has been predominately concerned with measuring and classifying an operator's functional states. Only recently has the field begun researching mitigation strategies. The purpose of this paper is to add further conceptual understanding to mitigation strategies. Based upon the decision making literature, we pose three issues that mitigation strategies need to resolve: the types of decision strategies an operator uses, the structure of the information that an operator processes, and finally, the cue or pattern of cues that the operator relies on in making decisions. These issues are important to ensure that mitigation strategies are congruent to operator's decision-making behaviors.
\end{abstract}

\section{INTRODUCTION}

Traditional approaches in human-computer interaction $(\mathrm{HCI})$ to alleviate human cognitive limitations have focused primarily on design and training [1]. For example, memory aids such as checklists or mnemonics are embedded within the design process and plant procedures to circumvent human memory limitations. Repetitive training and rotelearning are performed to improve performance under stressful conditions. And finally, task analyses are conducted during the design process to determine shared resources among operators to reduce workload. The major limitation to these approaches besides high costs and time is that they are ineffective in generalizing to unexpected events [1]. For instance, during an unanticipated event, automated control may be shifted to operator's manual control. This shift in control abruptly places high workload upon the operator. Under these situations, it is advantageous to have a system (similar to Figure 1) that can monitor, assess, and be sensitive to these human conditions.

For these reasons, developing a human augmented system (akin to physical system monitoring) that can actively monitor and assess an operator's level of workload and stress is essential to reduce plant risk (See Figure 1). The diagram in Figure 1 begins with a command (e.g., an event induces the control to be switch to manual) influencing the task. The sensors (e.g., physiological or behavioral gauges such as eyetracking and EEGs; see Reference [3] for a more detail discussion on different types of augmented system sensors) detect activities from the user, environment, and task, and produce models that reflect the current state of the user, environment, and task. The resulting models are integrated and interpreted by an augmentation manager that assesses and determines an appropriate strategy to mitigate the information system bottle-necks [2]. Hence, with an augmented cognition system, an operator's ongoing experience of heavy taskload, stress, or fatigue can be assessed by the system. The system can then re-configure and decrease the flowrate of information to heavy-tasked operator, guide the operator's attention to relevant information in the display by highlighting, blinking, or zooming in to the pertinent information [2], or the system can switch more of the workload activity away from the operator.

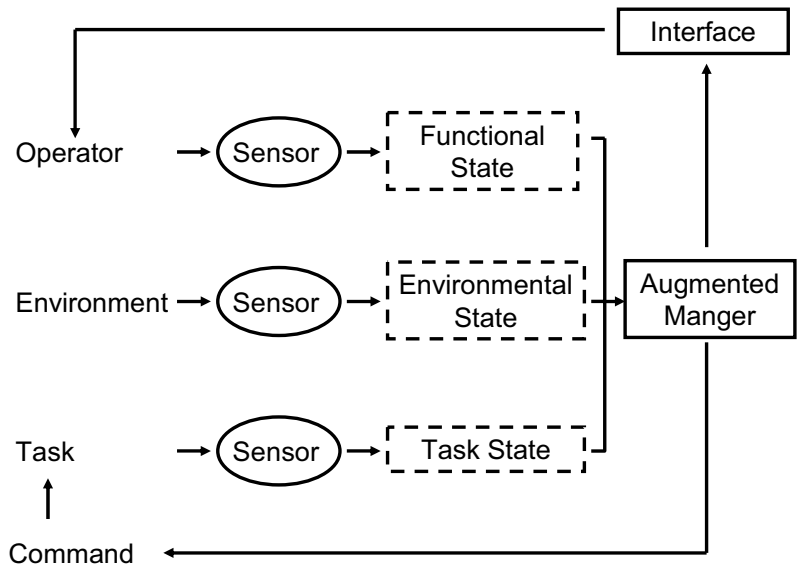

Figure 1. Diagram of An Augmented System (adapted from Reference [2])

Thus, the purpose of mitigation strategies is to reestablish optimal performance through manipulation of the work environment [4]. An analogy is an air conditioning system that is triggered, when the thermostat senses the room temperature is high, to reestablish the ideal room temperature. The assumption of an augmented system is that human informationprocessing bottlenecks (e.g., amount of information that can be attended and processed within a certain time) can be circumvented or overcome through use of effective mitigation strategies [5]. Research has demonstrated that effective use of mitigation strategies can improve operator performance, enhance situation awareness, and reduce performance errors [4].

One common mitigation strategy is task sequences, determining the appropriate scheduling, ordering, or prioritizing of information presented to the operator $[4$, 
5]. For example, when the augmented system detects a high level of workload from the operator, the augmented system could reduce information load by presenting fewer and higher priority information while keeping low priority information on hold until the augmented system determines that the high workload has been resolved. A limitation of task sequences is that the operator does not have access to all information [4]. Because of this, a task sequence mitigation strategy needs to be designed within the theories of human decision making to avoid operator's losing control his/her environment. This paper will address the following key issues relating to task sequence mitigation strategy:

- Decision Strategies - Accidentally altering an operator's decision strategies as when the task sequence invoking a more intuitive or heuristic processing when the situation calls for more elaborate problem-solving behaviors.

- Information Complexity - Consider information complexity in addition to scheduling and prioritizing of information such as when the situation calls for a quick sense-and-respond behavior but the information is too complex to support such behavior.

- Information Search Strategies - Disruption of the operator's information search strategy such as when the low priority information is a key element in a pattern-matching search strategy.

\section{Decision Strategies}

A number of theoretical models [6, 7, 8,] have suggested that operators use different decision making strategies to cope with the complexity of a dynamic environment - intuitive and analytical. Table 1 presents characteristics of intuition and analytical processes [9].

For instance, Hammond's [6] Cognitive Continuum Theory (CCT) places the decision processes in a continuum with one end of the continuum representing a fast, effortless, associative intuitive processing where the environmental cues are simply sensed and automatically responded. On the other end of the continuum is a slower serial and effortful analytical reasoning processing where environmental cues are integrated, interpreted, and evaluated. Finally, between the extreme ends in the continuum is the quasi-rational processing which represents a compromise between intuition and analytical processes. Quasi-rational processing occurs when full analysis of the situation is not possible and full intuition would not be acceptable [10].

This concept of dual-processes of decision making has recently been extended to encompassed experts' decision making performances. According to Klein's [7] Recognition-Primed Decision Making (RPD), experts have a wealth of knowledge (experiences) in their domain of expertise. Thus, experts can simply recognize a pattern of cues (i.e., pattern matching) or identify an important cue (i.e., pattern discrimination) to retrieve the relevant course of action(s) from memory. This pattern-matching behavior is believed to correspond to Hammond's notion of intuition processing, because it's fast and effortless. On the other hand, if a situation arises for which the expert is unsure of the proper course of action, experts would assess and evaluate the situation through "mental simulations" in which the expert answers the question, "If I do this, what is likely to happen?" [11]. Mental simulation is believed to correspond to Hammond's notion of analytical processing, because mental simulation takes time and effort, and is controlled.

TABLE 1. CHARACTERISTICS OF INTUITIVE AND ANALYTICAL PROCESSES

\begin{tabular}{|c|c|}
\hline $\begin{array}{c}\text { Intuition } \\
\text { (Pattern-Matching) }\end{array}$ & $\begin{array}{c}\text { Analytical } \\
\text { (Mental Simulation) }\end{array}$ \\
\hline Fast & Slow \\
\hline Parallel & Serial \\
\hline Automatic & Controlled \\
\hline Effortless & Effortful \\
\hline Associative & Rule-Governed \\
\hline Emotional & Neutral \\
\hline
\end{tabular}

A critical assumption in the dual-process decisionmaking framework is that optimal performances depend on successful mapping between the operator's decisionmaking strategies and the environment demand. An example would be quickly deciding on a good-enough solution based upon one's previous experience under high time pressure. On the other hand, sub-optimal performance occurs when the operator's decision strategies are ill-mapped in relation to the environment demand, for instance, evaluating a situation through mental simulations when the situation calls for an immediate response.

Because of this, it is argued here that mitigation strategies should be designed within the decisionmaking framework to ensure that the mitigation strategy is not inappropriately matched with the operator's decision strategies. For instance, during normal conditions in a nuclear power plant control room, an operator is occupied with monitoring behaviors. The operator's monitoring behavior during nominal conditions is complex, knowledge-driven, cognitively demanding, and can be characterized as a more problem-solving and planning activity than a passive vigilance activity [12]. To successfully assess system state and develop plans for anticipated future events, the operator requires assessing and evaluating a broad range of information to analytically simulate "what-if scenarios" as well as anticipatory plans if the plant state changes to off-normal. Thus, normal operating 
TABLE 2. OVERLAPPING LEVELS BETWEEN SRK, RPD, AND CCT

\begin{tabular}{|l|l|l|l|}
\hline Models & \multicolumn{3}{|c|}{ Decision-Making Levels } \\
\hline SRK & Skill-Based & Rule-Based & $\begin{array}{l}\text { Knowledge- } \\
\text { Based }\end{array}$ \\
\hline RPD & $\begin{array}{l}\text { Pattern } \\
\text { Matching }\end{array}$ & $\begin{array}{l}\text { Pattern } \\
\text { Discrimination }\end{array}$ & $\begin{array}{l}\text { Mental } \\
\text { Simulation }\end{array}$ \\
\hline CCT & Intuitive & $\begin{array}{l}\text { Quasi } \\
\text { Rationality }\end{array}$ & Analytical \\
\hline
\end{tabular}

conditions require that the operator uses analytical processes. Augmented systems that are insensitive to this need can accidentally disrupt the operator's decision making processes. For example, the augmented manager monitors and concludes that the operator is fatigued and initiates the task sequence mitigation strategy. The task sequence strategy shows only high priority information, thus disrupting the operator's ability to fully analyze the system state and anticipate future events. Furthermore, by presenting only partial information, task sequences may steer the operator to a more intuitively pattern-matching decision process (response based upon recognized cues) than an analytical mental simulation process of forecasting future plant states.

\section{INFORMATION COMPLEXITY}

Mitigation strategies also need to be sensitive to the appropriate level of information complexity that is best suited for different decision making processes. For this, a description of Rasmussen's [13] Skills-, Rules-, and Knowledge-Based (SRK) framework is needed.

Rasmussen labeled the different levels of cognitive control as skill-based (SB), rule-based (RB), and knowledge-based (KB). The skill-based (SB) level represents a highly experienced operator whose response tends to be automatic in that he or she doesn't need to interpret and integrate information but, rather, respond based upon relevant environmental cue(s) or "signals" to guide the action. The rule-based (RB) level represents a familiar operator who understands different rules (e.g., procedures) to use under different environmental conditions. Thus, the operator needs to interpret the environmental context or "signs" to apply the appropriate rules of action. Finally, at the knowledge-based (KB) level, the operator is not knowledgeable of the situation and, thus, relevant environmental cues (i.e., signals) as well as familiar context (i.e., signs) is absent. Because of this, the operator would need to integrate and interpret the environmental context or "symbols" more fully to select an appropriate action [11]. The SRK framework shares many similar features with the RPD and CCT frameworks and has since been attempted to be integrated in the literature (See Table 2; See Reference [10] for details).
An implication of the SRK framework is one that provides insights about the type of information that would be most suitable under different decision processes, "signals" in SB, to guide actions; "signs" in $\mathrm{RB}$ to trigger appropriate procedures and rules, and "symbols" in KB for information integration, interpretation, and evaluation. Because of this, it is argued here that mitigation strategies be designed to foster this mapping of information complexity to appropriate levels of decision-making processes. For example, during normal plant condition, the operator may want to process information complexity in the form of "symbols" to integrate and evaluate current and future plant conditions. When an off-normal event occurs, the operator may shift his or her attention to more of a rule-based or pattern discrimination decisionmaking processes in identifying the correct procedures or rules to follow to bring the plant back to a normal condition. During this time, the augmented system detects high arousal or workload and triggers task sequence mitigation strategies to limit the amount of information processing or highlights critical information sources. A mismatch between information complexity and decision-making processes occurs if the presented or highlighted information is still structured as "symbols," calling for higher decision-making strategies (i.e., knowledge-based) than what the operator is currently processing (i.e., rule-based). This mismatch would prevent operators from using rule-based processing and require them to shift to a higher knowledge-based processing that can put the system at risk if time is a factor.

\section{INFORMATION SEARCH}

Several theories of expert performance $[14,15,16]$ have discussed superior performance in terms of expert ability to identify relevant or meaningful subtle cues (or patterns of cues) in the environment to which they can exploit their rich and elaborative domain knowledge.

\section{The Lens Model (Brunswik)}

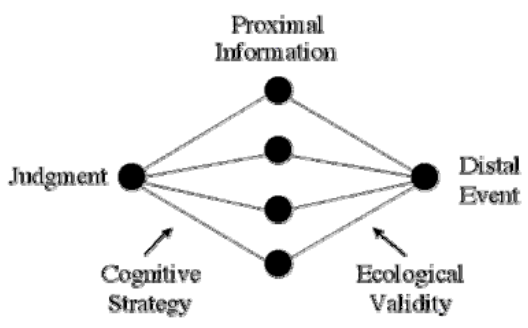

Figure 2. Diagram of Brunswick's Len Model 
This though is in line with Brunswik's [17] "Lens Model" (presented in Figure 2) in which information about the environment is represented by cues or patterns of cues (represented as proximal information in Figure 2). Superior expert performance emerges when experts can recognize these meaningful cues via their cognitive (information) search strategies to drive their decision making processes. As described by Vicente and Wang [14]:

"There can be expertise effects [in memory recall] when there are goal-relevant constraints (i.e., relationships pertinent to the domain) that experts can exploit to structure the stimuli. The more constraint available, the greater the expertise advantage can be. Fully random stimuli have no constraints, so no expertise advantage would be expected. To realize these potential advantages, experts must be attuned (i.e., they must attend) to the goal-relevant constraints in question. If they do not pick up on this information, then no expertise advantage is expected." (p. 36)

Because of this, it is argued here that the mitigation strategy is sensitive to what operators perceive in the environment as relevant or meaningful cues as well as ensuring that such cues are not blocked or patterns of cues are not distorted during the mitigation (e.g., task sequence). This is especially critical if the operator relies on such cues or patterns of cues to assist in deriving the correct procedure action or developing an elaborative understanding of the plant state. Thus, it is suggested that a clear understanding of what cue or pattern of cues the operator tends to use to diagnose and take action should be identified (via task analysis or abstraction hierarchy) during the design of mitigation strategies.

\section{CONCLUSION}

For a system akin to Figure 2 to be effective, it must be accurate in measuring the operator's functional state (i.e., workload), it must be accurate in classifying the operator's functional state (i.e., high vs low workload), and it must be capable of triggering appropriate mitigation strategies. Prior research on augmented systems has been predominately concerned with measuring and classifying the operator's functional states. Only recently has the field begun researching mitigation strategies and successfully discussed mitigation strategies in terms of how information should be presented (priority, highlighting, information flow), when should mitigation strategies be implemented without interrupting operator's primary task, and which modality (i.e., tactile, visual, auditory) the information should be presented based upon the working memory literature. The purpose of this paper was to add further conceptual understanding to mitigation strategies. Based on the decision making literature, we pose three issues that mitigation strategies would need to resolve: the types of decision strategies an operator use, the structure of the information that an operator processes, and finally, the cue or pattern of cues that the operator relies on in making decisions. These issues are important to ensure that mitigation strategies are congruent to operator's decision-making behaviors.

\section{DISCLAIMER}

This paper was prepared as an account of work sponsored by an agency of the United States Government. Neither the United States Government nor any agency thereof, nor any of their employees, makes any warranty, expressed or implied, or assumes any legal liability or responsibility for any third party's use, or the results of such use, of any information, apparatus, product, or process disclosed in this paper, or represents that its use by such third party would not infringe privately owned rights.

\section{REFERENCES}

[1] Pavel, M., Wang, G., \& Li, K. Augmented cognition: Allocation of attention. Proceedings of the $36^{\text {th }}$ Hawaii International Conference on System Sciences, 2002.

[2] Marshall, L., \& Raley, C. Platform-based design of augmented cognition systems. Available online at: http://www.isr.umd.edu/ austin/ense623.d/projects04.html.

[3] Tran, T.Q., Boring, R.L., Dudenhoeffer, D.D., Hallbert, B.P., Keller, D.M., \& Anderson, T.M. Advantages and disadvantages of physiological assessment for next generation control room design. Joint $8^{\text {th }}$ Annual IEEE Conference on Human Factors and Power Plants and $13^{\text {th }}$ Annual Workshop on Human Performance/Root Cause/Trending/Operating Experience/SelfAssessment, in press.

[4] Regli, S.H., Tremoulet, P., Hastie, H., \& Stibler, K. Mitigation strategy design for optimal augmented cognition systems. Proceedings of the 2nd Annual Augmented Cognition, 2006.

[5] Schmorrow, D., Stanney, K.M, Wilson, G., \& Young, P. Augmented cognition in human-system interaction. In G. Salvendy (Eds.), Handbook of Human Factors and Ergonomic $3^{\text {rd }}$ Edition, New Jersey: John Wiely \& Sons, 2006, pp. 1364 1384.

[6] Hammond, K.R. Introduction to Brunswikian theory and methods. In K.R. Hammond andN.E. Wascoe (Eds.), Realizations of Brunswik's experimental design. San Francisco: Jossey-Bass, 1980.

[7] Klein, G. A recognition-primed decision (RPD) model of rapid decision-making. In G.Klein, J. Orasanu, R. Calderwood, and C.E. Zsambok (Eds.), Decision-making in action:Models and methods (pp. 138-147). Norwood, NJ: Ablex, 1993.

[8] Stanovich, K. E., \& West, R. F. Individual differences in reasoning: Implications for the rationality debate. In T. Gilovich, D. Griffin, \& D. Kahneman (Eds.), Heuristics and biases (pp. 421-440). New York: Cambridge University Press, 2002.

[9] Kahneman, D. A perspective on judgment and choice: Mapping bounded rationality. American Psychologist, 58, 2003, pp. 697720 .

[10] Elgin, P.D., \& Thomas, R.P. An integrated decision-making model for categorizing weather products and decision aids. NASA/TM-2004-212990, 2004. 
[11] Wickens, C. D., Lee, J. D., Liu, Y., \& Gordon Becker, S. E., An Introduction to Human Factors Engineering, ( $2^{\text {nd }}$ Ed.), New Jersey, Prentice Hall, 2004.

[12] Mumaw, R.J., Roth, E.M., Vicente, K.J., \& Burns, C.M. There is more to monitoring a nuclear power plant than meets the eye. Human Factors, 41 (1), 2000, pp.36-55.

[13] Rasmussen, J. Skills, rules, knowledge: Signals, signs, and symbols and otherdistinctions in human performance models. IEEE Transactions on Systems, Man, and Cybernetics, 13(3),1983, pp. 257-267.

[14] Vicente, K. J., \& Wang, J. H. An ecological theory of expertise effects in memory recall. Psychological Review, 10 1998, 3357.

[15] Simon, H. A., \& Chase, W. G. Skill in chess. American Scientist, 61, 1973, 394-403.

[16] Gobet, F., \& Simon, H. A. Recall of rapidly presented random chess positions is a function of skill. Psychonomic Bulletin \& Review, 3, 1996b, 159-163.

[17] Brunswik, E. Perception and the representative design of psychological experiments (2nd ed.). Berkeley: University of California Press, 1956. 